\title{
EXTENSION OF THEOREMS BY KRASNOSELSKIJ, STEČENKO, DUGUNDJI, GRANAS, KIVENTIDIS, ROMAGUERA, CARISTI AND KIRK
}

\author{
Milan R. Tasković*
}

\begin{abstract}
In this paper we describe a class of conditions sufficient for the existence of a furcate point which generalize several known results of fixed point.
\end{abstract}

\section{Introduction and history}

In [6] Krasnoselskij and Stečenko investigated a mapping $T$ on a complete metric space $(X, \rho)$ that satisfies the following contiditon: there exist a continuous mapping $\Psi: \mathbb{R}_{+}^{0} \rightarrow \mathbb{R}_{+}^{0}:=[0,+\infty)$ such that

$$
\rho[T x, T y] \leq \rho[x, y]-\Psi(\rho[x, y]) \text { for all } x, y \in X,
$$

where $0<\Psi(t)<t$ for every $t \in \mathbb{R}_{+}:=(0,+\infty)$; and showed that such mappings have a unique fixed point in $X$.

Let $(X, \rho)$ be a metric space. A mapping $\theta: X \times X \rightarrow \mathbb{R}_{+}^{0}$ (not necessarily continuous) is called compactly positive on $X$, if

$$
\inf \{\theta(x, y): \alpha \leq \rho(x, y) \leq \beta\}>0
$$

for each finite closed interval $[\alpha, \beta] \subset \mathbb{R}_{+}$. In a former paper Dugundji and Granas [2] investigated a mapping $T$ on a complete metric space $(X, \rho)$ that satisfies the following condition: there exist a compactly positive $\theta$ on $X$ such that

$$
\rho[T x, T y] \leq \rho[x, y]-\theta(x, y) \quad \text { for all } x, y \in X,
$$

AMS (MOS) Subject Classification 1991. Primary: 47H10, 05A15. Secondary: 54H25.

Key words and phrases: Fixed point theorems, topological spaces, weakly contractive mappings, general monotone principle of fixed point, furcate points, monotone and forks principle.

*Research supported by Science Fund of Serbia under Grant 1457. 
and showed that such mappings have a unique fixed point in $X$. A mapping $T: X \rightarrow X$ satisfying (DG) is referred to as weakly contractive.

In recent papers Caristi [1] and Kirk [3] investigated a mapping $T$ on a complete metric space $(X, \rho)$ that satisfies the following condition: there exist a lower semicontinuous function $G: X \rightarrow \mathbb{R}_{+}^{0}$ such that

$$
\rho[x, T x] \leq G(x)-G(T x) \text { for every } x \in X,
$$

and showed that such mappings have a fixed point in $X$.

In this paper, we extend the preceding results of Krasnoselskij, Stečenko, Dugundji, Granas, Kiventidis, Romaguera, Tasković, Caristi, Kirk, also, and many others; and we describe a class of conditions sufficient for the existence of a furcate point which generalize several known results of fixed point.

\section{Main result}

In this section we introduce the concept of a "general monotone principle of forks point".

In connection with this, we shall introduce the concept of lower $B C S$ convergence in a space $X$ for $B: X \rightarrow \mathbb{R}_{+}^{0}$, i.e., a topological space $X$ satisfies the condition of lower BCS-convergence iff $\left\{a_{n}(x)\right\}_{n \in \mathbb{N}}$ is a sequence in $X$ with arbitrary $x \in X$ and if $B\left(a_{n}(x)\right) \rightarrow b=b(x) \geq 0(n \rightarrow \infty)$ implies that $\left\{a_{n}(x)\right\}_{n \in \mathbb{N}}$ has a convergent subsequence $\left\{a_{n(k)}(x)\right\}_{k \in \mathbb{N}}$ which converges to $\xi \in X$, where

$$
B(\xi) \leq \inf _{x \in X} \liminf _{k \rightarrow \infty} B\left(a_{n(k)}(x)\right) .
$$

We are now in a position to formulate our main statement (General monotone principle ) which generalize great numbers of known results.

Theorem 1. (Monotone and forks principle). Let $T$ be a mapping of a topological space $X$ into itself, where $X$ satisfies the condition of lower BCS-convergence. If

$$
B(T x) \leq B(x) \quad \text { for every } x \in X,
$$

then there exists a furcate point $\xi \in X$, i.e., such that

$$
B(T \xi)=B(\xi)=\alpha:=\inf _{x \in X} \lim _{n \rightarrow \infty} B\left(b_{n}(x)\right)
$$

for some sequence $\left\{b_{n}(x)\right\}_{n \in \mathbb{N}}$ in $X$ which converges to $\xi$.

We notice that in the case of the preceding statement, if $B(T x)=B(x)$ implies $T x=x$, then $T$ has a fixed point in $X$.

A brief proof of this statement based on some elementary facts may be found in: Tasković [9] and [10]. For this, also, see and: Tasković [11].

An immediate consequence of the preceding statement is the following result. 
Corollary 1. Let $T$ be a mapping of a topological space $X$ into itself with the property $(B)$ where $B: X \rightarrow \mathbb{R}_{+}^{0}$ is lower semicontinuous. If for arbitrary $x \in X$ the sequence $\left\{T^{n} x\right\}_{n \in \mathbb{N}}$ has a convergent subsequence, then there exists a point $\xi \in X$ such that $(M)$.

Proof of Theorem 1. Let $x$ be an arbitrary point in $X$. Then from (B) we have the following inequalities

$$
0 \leq \cdots \leq B\left(T^{n+1} x\right) \leq B\left(T^{n} x\right) \leq \cdots \leq B(T x) \leq B(x)
$$

for every $n \in \mathbb{N} \cup\{0\}$ and for every $x \in X$. Thus, for the sequence $\left\{B\left(T^{n} x\right)\right\}_{n \in \mathbb{N}}$, we obtain $B\left(T^{n} x\right) \rightarrow b \geq 0(n \rightarrow \infty)$ with arbitrary $x \in X$. This implies (from lower $B C S$-convergence) that its sequence $\left\{T^{n} x\right\}_{n \in \mathbb{N}}$ contains a convergent subsequence $\left\{T^{n(k)} x\right\}_{k \in \mathbb{N}}$ with limit $\xi \in X$. Since $X$ satisfies the condition of lower $B C S$-convergence, from (1), we have

$$
\begin{gathered}
\alpha:=\inf _{x \in X} \lim _{n \rightarrow \infty} B\left(T^{n} x\right) \leq \lim _{n \rightarrow \infty} B\left(T^{n} \xi\right) \leq \cdots \leq B\left(T^{n} \xi\right) \leq \cdots \\
\leq B(T \xi) \leq B(\xi) \leq \inf _{x \in X} \liminf _{k \rightarrow \infty} B\left(T^{n(k)} x\right)=\alpha,
\end{gathered}
$$

i.e., $B(T \xi)=B(\xi)=\alpha$. This means that $(\mathrm{M})$ holds, where the existing sequence $\left\{b_{n}(x)\right\}_{n \in \mathbb{N}}$, de facto, the preceding subsequence of the sequence of iterates $\left\{T^{n(k)} x\right\}_{k \in \mathbb{N}}$. If $B(T \xi)=B(\xi)=\alpha$ implies that $T \xi=\xi$, then $\xi \in X$ is a fixed point of $T$. The proof is complete.

\section{Applications}

We now show that the following statements are all special cases of the above Theorem 1.

Corollary 2. (Krasnoselskij-Stečenko [6]). Let $T$ be a self-map on a complete metric space $(X, \rho)$. Suppose that there exists a continuous function $\Psi: \mathbb{R}_{+}^{0} \rightarrow \mathbb{R}_{+}^{0}$ satisfying $0<\Psi(t)<t$ for $t>0$ such that

$$
\rho[T x, T y] \leq \rho[x, y]-\Psi(\rho[x, y]) \quad \text { for all } x, y \in X,
$$

then $T$ has a unique fixed point $\xi \in X$, and at this point $T$ is continuous.

This statement is a special case of more general result obtained by Kiventidis [5]. This is analogous to the proof of the preceding statement.

Corollary 3. (Kiventidis [5]). Let $X$ be a Hausdorff space, $T: X \rightarrow X$ a continuous mapping, and $F: X \times X \rightarrow \mathbb{R}_{+}^{0}$ be a continuous function such that $F(x, y) \neq 0$ for all $x, y \in X(x \neq y)$ and

$$
F(T x, T y) \leq F(x, y)-\Psi(F(x, y)) \quad \text { for all } x, y \in X,
$$

where $\Psi: \mathbb{R}_{+}^{0} \rightarrow \mathbb{R}_{+}^{0}$ is a continuous function with property $0<\Psi(r)<r$ for every $r \in \mathbb{R}_{+}$. If for some $x \in X$ the sequence of iterates $\left\{T^{n} x\right\}_{n \in \mathbb{N}}$ has a convergent subsequence, then $T$ has a unique fixed point in $X$. 
We notice, Corollary 3 is an extension of result by Krasnoselskij-Stečenko [6] in case of metric space $(X, \rho)$ for $F(x, y):=\rho[x, y]$, obtained by Kiventidis [5]. Thus we have only to show this result.

Proof of Corollary 3. Let $B(x)=F(x, T x)$ which is lower semicontinuous on $X$, then for $y=T x$ from (K) we obtain (B) in Theorem 1. Since $X$ satisfies the condition of lower $B C S$-convergence $\left(\left\{T^{n} x\right\}_{n \in \mathbb{N}}\right.$ has a convergent subsequence which convergs to $\xi \in X)$, applying Theorem 1 we obtain $B(T \xi)=B(\xi)=\alpha$. Thus, from (K) we have $\alpha \leq \alpha-\Psi(B(\xi))$, i.e., we have a contradiction, which means that $T \xi=\xi$ for some $\xi \in X$. Uniqueness follows immediately from condition (K). The proof is complete.

Corollary 4. (Dugundji-Granas [2]). Let $(X, \rho)$ be a complete metric space, and $T: X \rightarrow X$ weakly contractive:

$$
\rho[T x, T y] \leq \rho[x, y]-\theta(x, y) \quad \text { for all } x, y \in X,
$$

where $\theta$ is compactly positive on $X$. Then $T$ has a unique fixed point $\xi$ in $X$, and at this point $T$ is continuous.

This result is a special case of the following more general result of type a locally form of (DG) obtained by Taskovic [8]. This has been generalized by Romaguera [7].

Corollary 5. (Tasković $[8])$. Let $(X, \rho)$ be a complete metric space and $g: X \rightarrow X$ an arbitrary mapping. Suppose for every $x \in X$ that is

$$
\rho\left[g(x), g^{2}(x)\right] \leq \rho[x, g(x)]-\theta(x, g(x)),
$$

where $\theta$ is compactly positive on $X$. If $x \mapsto \rho[x, g(x)]$ is lower semicontinuous, then $g$ has a fixed point in $X$.

Proof. Let $B(x)=\rho[x, g(x)]$ which is lower semicontinuous on $X$, then from (T) we obtain (B) in Theorem 1. Also, (T) implies $\rho\left[g^{n}(x), g^{n+1}(x)\right] \rightarrow 0$ $(n \rightarrow \infty)$ and, since $X$ is a complete metric space we have (see the Lemma of Dugundji-Granas [2, p. 142]) that $\left\{g^{n}(x)\right\}_{n \in \mathbb{N}}$ converges to some $\xi \in X$, i.e., $X$ that satisfies the condition of lower $B C S$-convergence. Hence, from Theorem 1 it follows $B(g(\xi))=B(\xi)=\alpha$. Thus, from (T) we obtain $\alpha \leq$ $\alpha-\theta(\xi, g(\xi))$, i.e., we have a contradiction, which means that $g(\xi)=\xi$ for some $\xi \in X$. The proof is complete.

Corollary 6. (Caristi [1], Kirk [3]). Let $T$ be a self-map on a complete metric space $(X, \rho)$. Suppose that there exists a lower semicontinuous function $G$ of $X$ into $\mathbb{R}_{+}^{0}$ such that

$$
\rho[x, T x] \leq G(x)-G(T x) \quad \text { for every } x \in X,
$$

then $T$ has a fixed point $\xi$ in $X$. 
Proof. Let $B(x)=G(x)$ which is lower semicontinuous on $X$, then $B(T x) \leq B(x)$ for every $x \in X$, i.e., (B) in Theorem 1 . Since $X$ satisfies the condition of lower BCS-convergence $(X$ is a complete metric space and for $x_{n}:=T^{n}(x)$ from $(\mathrm{CK})$ we have

$$
\sum_{i=0}^{\infty} \rho\left[x_{i}, x_{i+1}\right] \leq B(x),
$$

i.e., $B\left(T^{n} x\right) \rightarrow b \geq 0(n \rightarrow \infty)$ implies that $\left\{T^{n} x\right\}_{n \in \mathbb{N}}$ converges to some $\xi \in X)$, applying Theorem 1 we obtain $B(T \xi)=B(\xi)=\alpha$. Thus from (CK) we obtain $\rho[\xi, T \xi] \leq \alpha-\alpha$, i.e., $\xi=T \xi$ for some $\xi \in X$. The proof is complete.

In connection with the preceding, from the proof of Theorem 1, we obtain, as a directly extension of Theorem 1, the following general result.

Theorem 2. Let $T$ be a mapping of a topological space $X$ into itself, where $X$ satisfies the condition of lower BCS-convergence. If

$$
B(T x) \leq B(x) \quad \text { for every } \quad x \in X,
$$

then there exists an $k$-furcate point $\xi \in X$, i.e., such that

$$
B\left(T^{k} \xi\right)=\cdots=B(T \xi)=B(\xi)=\alpha:=\inf _{x \in X} \lim _{n \rightarrow \infty} B\left(b_{n}(x)\right),
$$

for arbitrary fixed integer $k \geq 1$ and for some sequence $\left\{b_{n}(x)\right\}_{n \in \mathbb{N}}$ in $X$ which converges to $\xi$.

We notice that in the preceding case of the statement, if $(\mathrm{Mk})$ implies $\xi=T \xi$, then $T$ has a fixed point in $X$.

Proof of this statement is a totally analogous with the proof of Theorem 1. Precision, from the proof of Theorem 1, directly we obtain Theorem 2.

As an immediate consequence of this result we obtain the following generalization of Caristi and Kirk's fixed point theorem.

Corollary 7. (Tasković [12]). Let T be a self-map on a complete metric space $(X, \rho)$. Suppose that there exist a lower semicontinuous function $G$ : $X \rightarrow \mathbb{R}_{+}^{0}$ and an arbitrary fixed integer $k \geq 0$ such that

$$
\rho[x, T x] \leq G(x)-G(T x)+\cdots+G\left(T^{2 k} x\right)-G\left(T^{2 k+1} x\right)
$$

and $G\left(T^{2 i+1} x\right) \leq G\left(T^{2 i} x\right)$ for $i=0,1 \ldots, k$ and for every $x \in X$. Then $T$ has a fixed point $\xi$ in $X$.

Proof. Let $x$ be an arbitrary point in $X$. We can show then that the sequence of iterates $\left\{T^{n} x\right\}_{n \in \mathbb{N}}$ is a Cauchy sequence. Let $n$ and $m(n<m)$ be any positive integers. From (Tk) we have

$$
\rho\left[T^{n} x, T^{m} x\right] \leq \sum_{i=n}^{m-1} \rho\left[T^{i} x, T^{i+1} x\right] \rightarrow 0 \quad(m, n \rightarrow \infty) .
$$


Hence $\left\{T^{n} x\right\}_{n \in \mathbb{N}}$ is a Cauchy sequence in $X$ and, by completeness, there is $\xi \in X$ such that $T^{n} x \rightarrow \xi(n \rightarrow \infty)$. Therefore, $X$ satisfies the condition of lower BCS-convergence for $B(x)=G(x)$.

Also, we have $B(T x) \leq B(x)$ for every $x \in X$, i.e., (B) in Theorem 2 . Applying Theorem 2 we obtain a form of $(\mathrm{Mk})$, i.e., $B\left(T^{2 k+1} \xi\right)=B\left(T^{2 k} \xi\right)=$ $\cdots=B(T \xi)=B(\xi)=\alpha$ for some $\xi \in X$. Thus from (Tk) we have

$\rho[\xi, T \xi] \leq G(\xi)-G(T \xi)+\cdots+G\left(T^{2 k} \xi\right)-G\left(T^{2 k+1} \xi\right)=(2 k+1)(\alpha-\alpha)=0$, i.e., $\xi=T \xi$ for some $\xi \in X$. The proof is complete.

Remark. We notice that a brief proof of Corollary 7 based on the Zorn's Lemma may be found in Tasković [12].

Theorem 3. Let $T$ be a mapping of a topological space $X$ into itself, where $X$ satisfies the condition of lower BCS-convergence. If

$$
B(T x) \leq B(x) \quad \text { for every } x \in X,
$$

then there exists an $\boldsymbol{m}(\boldsymbol{k})$-furcate point $\xi \in X$, i.e., such that

$$
B\left(T^{m} \xi\right)=\cdots=B\left(T^{k} \xi\right)=\cdots=B(T \xi)=B(\xi)=\alpha:=\inf _{x \in X} \lim _{n \rightarrow \infty} B\left(b_{n}(x)\right)
$$

for some fixed integer $k \geq 1$, for arbitrary integer $m \geq k$ and for some sequence $\left\{b_{n}(x)\right\}_{n \in \mathbb{N}}$ in $X$ which converges to $\xi$.

Proof of this statement is a totally analogous with the proof of Theorems 1 and 2 .

We notice that in the preceding case of the statement, if $(\mathrm{Mm})$ implies $\xi=T \xi$, then $T$ has a fixed point in $X$.

As an immediate consequence of the preceding result we obtain the following statement of fixed point.

Corollary 8. Let $T$ be a self-map on a complete metric space $(X, \rho)$. Suppose that there exists a lower semicontinuous function $G: X \rightarrow \mathbb{R}_{+}^{0}$ such that

$$
\rho[x, T x] \leq \sum_{i=0}^{+\infty}\left(G\left(T^{2 i} x\right)-G\left(T^{2 i+1} x\right)\right)
$$

and $G\left(T^{2 i+1} x\right) \leq G\left(T^{2 i} x\right)$ for $i \in \mathbb{N} \cup\{0\}$ and for every $x \in X$. Then $T$ has a fixed point $\xi$ in $X$.

Proof. Let $x$ be an arbitrary point in $X$. Then, from the proof of Corollary 7 , we obtain that $\left\{T^{n} x\right\}_{n \in \mathbb{N}}$ is a Cauchy sequence in $X$ and, by completeness, there is $\xi \in X$ such that $T^{n} x \rightarrow \xi(n \rightarrow \infty)$. Therefore, $X$ satisfies the condition of lower BCS-convergence for $B(x)=G(x)$. 
Also we have $B(T x) \leq B(x)$ for every $x \in X$, i.e., (B) in Theorem 3 . Applying Theorem 3 we obtain a form of $(\mathrm{Mm})$, i.e., we have

$$
B\left(T^{2 m+1} \xi\right)=B\left(T^{2 m} \xi\right)=\cdots=B\left(T^{k} \xi\right)=\cdots=B(T \xi)=B(\xi)=: \alpha
$$

for some $\xi \in X$ and integer $k \geq 1$ and for every integer $m \geq k$. Thus, from $(\mathrm{Tm})$, we have

$$
\begin{gathered}
\rho[\xi, T \xi] \leq \sum_{i=0}^{+\infty}\left(G\left(T^{2 i} \xi\right)-G\left(T^{2 i+1} \xi\right)\right)= \\
=\sum_{i=0}^{m}\left(G\left(T^{2 i} \xi\right)-G\left(T^{2 i+1} \xi\right)\right)+ \\
+\sum_{i=m+1}^{+\infty}\left(G\left(T^{2 i} \xi\right)-G\left(T^{2 i+1} \xi\right)\right)= \\
=(m+1)(\alpha-\alpha)+\sum_{i=m+1}^{+\infty}\left(G\left(T^{2 i} \xi\right)-G\left(T^{2 i+1} \xi\right)\right)= \\
=0+\sum_{i=m+1}^{+\infty}\left(G\left(T^{2 i} \xi\right)-G\left(T^{2 i+1} \xi\right)\right),
\end{gathered}
$$

therefore, as $m \rightarrow+\infty$, we obtain $\rho[\xi, T \xi] \leq 0$, i.e., $\xi=T \xi$ for some $\xi \in X$. The proof is complete.

\section{Some new geometric theorems}

As further applications of the preceding Theorems 1, 2 and 3 we obtain the following geometric statements of fixed point on complete metric spaces.

Theorem 4. Let $T$ be a self-map on a complete metric space $(X, \rho)$. Suppose that there exists a lower semicontinuous function $G: X \rightarrow[a,+\infty)$ for some $a>0$ such that

$$
\rho[x, T x] \leq \frac{1}{G(T x)}-\frac{1}{G(x)} \quad \text { for every } x \in X,
$$

then $T$ has a fixed point $\xi$ in $X$.

Proof of this statement is an analogous to the proof of Corollary 6 based on Theorem 1.

As a directly extension of Theorem 4, from Theorem 2, we obtain the following result of fixed point on complete metric spaces. 
Theorem 5. Let $T$ be a self-map on a complete metric space $(X, \rho)$. Suppose that there exist a lower semicontinuous function $G: X \rightarrow[a,+\infty)$ for some $a>0$ and an arbitrary fixed integer $k \geq 0$ such that

$$
\rho[x, T x] \leq \frac{1}{G(T x)}-\frac{1}{G(x)}+\cdots+\frac{1}{G\left(T^{2 k+1} x\right)}-\frac{1}{G\left(T^{2 k} x\right)}
$$

and $G\left(T^{2 i+1} x\right) \leq G\left(T^{2 i} x\right)$ for $i=0,1, \ldots, k$ and for every $x \in X$. Then $T$ has a fixed point $\xi$ in $X$.

We notice that for $k=0$ in Theorem 5 we have Theorem 4. Proof of this statement is based on Theorem 2 .

Proof of Theorem 5. Let $x$ be an arbitrary point in $X$. We can show then that the sequence $\left\{T^{n} x\right\}_{n \in \mathbb{N}}$ is a Cauchy sequence. Let $n$ and $m(n<m)$ be any positive integers. From the property (Rk), we have

$$
\sum_{i=0}^{n} \rho\left[T^{i} x, T^{i+1} x\right] \leq \frac{1}{G\left(T^{n+1} x\right)}-\frac{1}{G(x)},
$$

and thus, since $a<G\left(T^{n+1} x\right)$ for every $x \in X$ and for every $n \in \mathbb{N} \cup\{0\}$, we obtain the following fact:

$$
\rho\left[T^{n} x, T^{m} x\right] \leq \sum_{i=n}^{m-1} \rho\left[T^{i} x, T^{i+1} x\right] \rightarrow 0 \quad(n, m \rightarrow \infty) .
$$

Hence $\left\{T^{n} x\right\}_{n \in \mathbb{N}}$ is a Cauchy sequence in $X$ and, by completeness, there is $\xi \in X$ such that $T^{n} x \rightarrow \xi(n \rightarrow \infty)$. Therefore, $X$ satisfies the condition of lower BCS-convergence for $B(x)=G(x)$ and the condition (B) in Theorem 2. Applying Theorem 2, we obtain a form of (Mk), i.e., $B\left(T^{2 k+1} \xi\right)=\cdots=$ $B(T \xi)=B(\xi)=\alpha$, for some $\xi \in X$. Thus, from the property (Rk), we have

$$
\begin{gathered}
\rho[\xi, T(\xi)] \leq \frac{1}{G(T \xi)}-\frac{1}{G(\xi)}+\cdots+\frac{1}{G\left(T^{2 k+1} \xi\right)}-\frac{1}{G\left(T^{2 k} \xi\right)}= \\
=(2 k+1)\left(\frac{1}{\alpha}-\frac{1}{\alpha}\right)=0,
\end{gathered}
$$

i.e., $\xi=T \xi$ for some $\xi \in X$. The proof is complete.

In connection with the preceding, as a directly consequence of Theorem 3 , we obtain the following statement of fixed point on complete metric spaces.

Theorem 6. Let $T$ be a self-map on a complete metric space $(X, \rho)$. Suppose that there exists a lower semicontinuous function $G: X \rightarrow[a,+\infty)$ for some $a>0$ such that

$$
\rho[x, T x] \leq \sum_{i=0}^{+\infty}\left(\frac{1}{G\left(T^{2 i+1} x\right)}-\frac{1}{G\left(T^{2 i} x\right)}\right)
$$

and $G\left(T^{2 i+1} x\right) \leq G\left(T^{2 i} x\right)$ for $i \in \mathbb{N} \cup\{0\}$ and for every $x \in X$. Then $T$ has a fixed point $\xi$ in $X$. 
Proof of this statement is a totally analogous with the proof of Corollary 8. Precision, from the preceding Theorem 3, directly we obtain Theorem 6 as a consequence.

\section{Further interpretation of the forks theorems}

We notice, in this part, that for the preceding main statements we can give their the following explanations via the BCS- completeness and the lower BCS-continuous in the following sense.

In connection with this, we shall introduce the concept of BCS-completeness in a space $X$ for a function $B: X \rightarrow \mathbb{R}$, i.e., a topological space $X$ is called BCS-complete iff $\left\{a_{n}(x)\right\}_{n \in \mathbb{N}}$ is a sequence in $X$ with arbitrary $x \in X$ and if $B\left(a_{n}(x)\right) \rightarrow b=b(x) \in \mathbb{R} \cup\{ \pm \infty\}$ as $n \rightarrow \infty$ implies that $\left\{a_{n}(x)\right\}_{n \in \mathbb{N}}$ has a convergent subsequence in $X$.

On the other hand, a function $B: X \rightarrow \mathbb{R}$ is lower BCS-continuous at $p \in X$ iff $\left\{a_{n}(x)\right\}_{n \in \mathbb{N}}$ is a sequence in $X$ with arbitrary $x \in X$ and if $a_{n}(x) \rightarrow p(n \rightarrow \infty)$ implies that is

$$
B(p) \leq \inf _{x \in X} \liminf _{n \rightarrow \infty} B\left(a_{n}(x)\right) .
$$

We are now in a position to formulate the following explanations of the preceding theorems as corresponding equivalent forms:

Theorem 1a. Let $T$ be a mapping of a topological space $X$ into itself and let $X$ be BCS-complete. If $(B)$ holds and if $B: X \rightarrow \mathbb{R}_{+}^{0}$ is a lower $B C S$-continuous functional, then there exists a furcate point $\xi \in X$.

Proof of this statement is a totally analogous with the proof of Theorem 1 which is equivalent to the Theorem 1a.

Theorem 2a. Let $T$ be a mapping of a topological space $X$ into itself, where $X$ satisfies the condition of BCS-completeness. If (B) holds and if $B: X \rightarrow \mathbb{R}_{+}^{0}$ is a lower BCS-continuous functional, then there exists an $k$-furcate point $\xi \in X$.

Also, as in the preceding case of Theorem 1a, proof of this statement is a totally analogous with the proof of Theorem 2 which is equivalent to the Theorem $2 \mathrm{a}$.

Theorem 3a. Let $T$ be a mapping of a topological space $X$ into itself and let $X$ be BCS-complete. If

$$
B(T x) \leq B(x) \text { for every } x \in X,
$$

and if $B: X \rightarrow \mathbb{R}_{+}^{0}$ is a lower BCS-continuous functional, then there exists an $m(k)$-furcate point $\xi \in X$, i.e., such that

$$
B\left(T^{m} \xi\right)=\cdots=B\left(T^{k} \xi\right)=\cdots=B(T \xi)=B(\xi)=\alpha:=\inf _{x \in X} \lim _{n \rightarrow \infty} B\left(b_{n}(x)\right)
$$


for some fixed integer $k \geq 1$, for arbitrary integer $m \geq k$ and for some sequence $\left\{b_{n}(x)\right\}_{n \in \mathbb{N}}$ in $X$ which converges to $\xi$.

We notice that the proof and of this statement, also, is a totally analogous with the proof of Theorem 3 which is equivalent to the Theorem $3 \mathrm{a}$.

\section{References}

[1] J. Caristi: Fixed point theorems for mappings satisfying inwardness conditions, Trans.Amer.Math. Soc., 215 (1976), 241-251.

[2] J. Dugundji and A. Granas: Weakly contractive maps and elementary domain invariance theorem, Bull.Greek.Math.Soc., 19 (1978), 141-151.

[3] W.A. Kirk: Caristi's fixed point theorem and metric convexity, Colloq. Math., 36 (1976), 81-86.

[4] W.A. Kirk and J. Caristi: Mapping theorems in metric and Banach spaces, Bull. Acad. Polon., 23 (1975), 891-894.

[5] T. Kiventidis: On fixed points in Hausdorff spaces, Indian J.Math., 16 (1985), 1420-1424.

[6] M. A. Krasnoselskij and V. Stečenko: K teorii uravnenia s vognutim operatorami, Sib.Math.J., 10(1969), 562-572.

[7] S. Romaguera: Fixed point theorems for mapping in complete quasi-metric space, Anal.Stii.Universit. "Al.I.Cuza" Iaşi, 39(1993), 159-164.

[8] M.R. Tasković: A monotone principle of fixed points, Proc.Amer.Math. Soc., 94(1985), 427-432.

[9] M. R. Tasković: Nonlinear Functional Analysis, Second Book: Monographs - Global Convex Analysis - General convexity, Variational methods and Optimization, Zavod za udžbenike i nastavna sredstva and Vojnoizdavački zavod, Beograd 2001, (in Serbian), 1223 pages.

[10] M.R. Tasković: Transversal and fixed points, forks, general convex functions and apllications, Monographs: Zavod za udžbenike i nastavna sredstva and Vojnoizdavački zavod, Beograd 2001, Nonlinear Functional Analysis, Vol. 2 (2001), 1067-1172.

[11] M. R. Tasković: General expansion mappings on topological spaces, Scientiae Math. Japonicae, 54, No.1 (2001), 61-67,: e4, 497-503.

[12] M. R. Tasković: New geometric fixed point theorems, Math. Moravica, 2(1998), 143-148.

Matematički fakultet, 11000 Beograd, P.O. Box 550, Yugoslavia

Received January 7, 2001. 